\title{
Fellows Column: Neonatal Abstinence Syndrome: Management and Role of APRN
}

Rachel Fiorentino, BSN

\begin{abstract}
:
Substance abuse is on the rise in the United States, and opioids have been the drug of choice. When pregnant women abuse opioids, the newborns can experience a set of symptoms known as neonatal abstinence syndrome (NAS). Screenings should be implemented with every prenatal visit to assist with a treatment program or support prior to birth. When screenings fail to assist, biological testing should be obtained to forecast withdrawal. Symptoms need to be assessed with a clinical diagnostic tool such as "Eat, Sleep, Console" and treated with non-pharmacological interventions first, and then pharmacological if unresolved. The advanced practice nurse is a key factor in coordinating this care and advancing public health policies.
\end{abstract}

Keywords: Neonatal abstinence syndrome, NAS, opioid epidemic, Eat, Sleep, Console

\section{"It has been hypothesized that neonatal abstinence syndrome (NAS) was a part of society before the 1900s. Nevertheless, the rate of opioid-addicted pregnant women is increasing exponentially. This brings a priority to define, screen, test, assess, and treat neonates withdrawing from opioids promptly and successfully"}

\section{Neonatal Abstinence Syndrome:}

The opioid epidemic has been a focus for public health, US Drug Enforcement, and health care providers for quite some time, with the first reported case in 1875 (1). It has been hypothesized that neonatal abstinence syndrome (NAS) was a part of society before the 1900s. Nevertheless, the rate of opioid-addicted pregnant women is increasing exponentially. This brings a priority to define, screen, test, assess, and treat neonates withdrawing from opioids promptly and successfully.

\section{Definition, Incidence, Presentation, and Physical Findings:}

The incidence of opioid usage during pregnancy has increased dramatically. Globally, usage has increased by $47 \%$ from $1990-$ 2016 (2). In the United States, there has been an increase of 333\% from 1999-2014(2). According to the Centers for Disease Control and Prevention (CDC), in 2016, one baby every 19 minutes was diagnosed with NAS (3). The states with the highest rates include Kentucky, New Mexico, Pennsylvania, Tennessee, Vermont, and West Virginia (3).

NAS is a collection of clinical signs and symptoms from abrupt discontinuation of opioids at birth after exposure in utero (4). The severity of symptoms varies based on length of exposure, type of drug, purity of drug, polysubstance use, and gestational age of the neonate (1). The substances can be prescribed or non-prescribed, one specific opioid or a combination of many. The most common maternal substance use is opioids such as OxyContin, fentanyl, heroin, and morphine. There is a high risk of polysubstance use with amphetamines, cocaine, cannabinoids, nicotine, alcohol, and benzodiazepines, along with opioids.

The range of clinical presentations for NAS can be divided into two categories: central nervous system and gastroenterology. The most common symptoms are "increased muscle tone, tremors when undisturbed, and exaggerated Moro reflex" (2). Other characteristics of NAS include excessive high-pitched crying, jitteriness, tremors, diarrhea, nasal stuffiness, sneezing, poor feeding, hypertonia, frequent yawning, diaphoresis, and temperature instability (4). These listed symptoms can continue to create additional complications that will also need to be managed. Examples include diarrhea leading to severe skin breakdown or poor feeding leading to poor weight gain and failure to thrive. While NAS is selflimiting, it can last from weeks to six months (5).

The most severe and rarely reported presentation of NAS is seizures (1). It is estimated that between $2-11 \%$ of NAS infants may have seizures (6). However, it is difficult to distinguish from observable seizure-like activity versus epileptic seizures demonstrated on electroencephalogram (EEG). A seizure-like activity might indicate benign neonatal sleep myoclonus (BNSM) self-limiting and not requiring treatment (6).

The onset of symptoms depends on the type of drug exposure in utero. Short half-life opioids such as heroin may have infants withdrawing within 24 hours (2). While long-acting opioids, such as buprenorphine and methadone, could take up to three days. There have been reports of opioid withdraw not occurring until 30 days of life. These time frames for clinical presentations can be more problematic with additional polysubstance use. Withdraw from benzodiazepines or barbiturates can take up to seven to 21 days to onset symptoms (2).

\section{Disease Etiology and Pathogenesis:}

Opioids cross through the placenta easily because of their fatloving or lipophilic properties (4). These lipophilic properties allow opioids to cross the blood-brain barrier and accumulate in fetal brains (4). Animal studies have shown a cascading effect on neurotransmitters, opioid receptors, locus coeruleus, and various intracellular elements with neonatal withdraw of an opioid at the time of birth (1). When the fetus is exposed to opioids, the receptors in the locus coeruleus increase intracellular cyclic adenosine monophosphate (cAMP), causing hyperactivity. When the opioids are withdrawn, the over-activation of cAMP causes an increase in norepinephrine release. With an overabundance of norepinephrine, the "flight-or-fight" response is on overdrive, causing central nervous system irritability and gastrointestinal dysfunction (1).

Levels of neurotransmitters begin to fluctuate, increasing central nervous system irritability (1). Dopamine and serotonin levels de- 
crease, which alters sleep, appetite, and stress. The accumulation of all these attributes, in addition to an immature nervous system, hinders the newborn's state control, sensory processing, and motor control (1).

\section{"Management of NAS begins with screening. Most, if not all, obstetricians ask routine questions regarding prenatal drug usage. This screening can open the door for women to seek treatment programs while pregnant. Unfortunately, reliability of answers, fear of judgment, and a tendency for other high-risk behaviors may not reveal the information needed until the symptoms} are withessed."

\section{Management:}

Management of NAS begins with screening. Most, if not all, obstetricians ask routine questions regarding prenatal drug usage. This screening can open the door for women to seek treatment programs while pregnant. Unfortunately, reliability of answers, fear of judgment, and a tendency for other high-risk behaviors may not reveal the information needed until the symptoms are witnessed. When that does become the case, caregivers need to know highrisk maternal signs for biological testing. These signs may include no prenatal care, sexually transmitted diseases, premature labor, and abruptio placentae (4). Hospitals should have policies regarding when to perform biological testing to avoid biases while still protecting the neonate.

Several types of biological testing can be done, each with pros and cons. Maternal and infant urine testing is quick and less expensive than other testing (2). However, there is a high probability of false negatives depending on the last use of the drug(s), dosage, and cumulative in-utero exposure. Meconium testing is more sensitive and can detect exposure from 20 weeks gestation to birth. Meconium testing requires strict protocols on handling and cannot be contaminated with urine, water, or alcohol. False positives can occur with amphetamines. Neither urine nor meconium can identify synthetic opioids, such as fentanyl, without it being specified. Cord blood and hair can detect drug use in utero but can be costly and require specialized experts. Many facilities will use urine with meconium verification (2).

In attempts to manage NAS, many models of care have been developed. These models strive to care for the infant as best as possible with as little pharmacological intervention as needed with the shortest length of stay (5). These models include an assessment tool to determine when treatment should be escalated to pharmacological interventions. The most recent assessment tool is Eat, Sleep, Console (ESC) (5). The guidelines are as follows: eat an appropriate amount daily, sleep undisturbed for at least one hour, and be consoled within 10 minutes (7). ESC navigates to- wards functional criteria of the newborn as opposed to responses to treatment (7).

When pharmacological intervention is needed, morphine is the most used first-line medication (2). According to Grossman \& Berkwitt (2019), 90\% of pharmacologically treated infants received morphine. Clonidine or phenobarbital is the most used second-line medication intervention. There are many studies to determine which medication has the best outcome for neonates, but there is currently no consensus (1).

\section{Outcomes:}

Whether non-pharmacologic or pharmacologic interventions are used, one key aspect continues to provide the best outcomes for the neonate. That is maternal involvement (5). Maternal bonding, attachment, breastfeeding, and skin-to-skin contact provide the infant with the best outcomes while mitigating NAS. However, maternal mental health should be acknowledged and supported to empower the mother to care for her child (5).

Studies of long-term outcomes of NAS are limited and lack precision causation (1). For example, children with NAS may have poorer school performance than their peers; but is that from low socio-economic factors, lack of parental involvement, or opioid use in utero? A New York study suggested children with NAS had a mortality rate four times greater but is that from high-risk behaviors associated with parental addiction or withdrawing from opioids as an infant? There is still more to be discovered about the long-term outcomes of NAS (1).

\section{"An advanced practice nurse (APN) can affect NAS and the opioid epidemic in three different ways. One, ensure correct care of the neonate. Two, publish findings or remain current on research. And three, help facilitate change policies as it relates to legislation. Care for the infant will always be the first and foremost implication for an APN."}

\section{Implications for Advanced Practice Nursing:}

An advanced practice nurse (APN) can affect NAS and the opioid epidemic in three different ways. One, ensure correct care of the neonate. Two, publish findings or remain current on research. And three, help facilitate change policies as it relates to legislation. Care for the infant will always be the first and foremost implication for an APN. This process includes utilizing assessment tools, optimizing non-pharmacological care, and implementing medications when warranted. The APN should also educate and support staff. It can be difficult for staff nurses to remain non-judgmental when caring for an infant with a high-pitched cry that requires constant holding. APN's need to consult other resources such as social workers to ensure holistic care. This treatment, when possible, should include the mother. The APN should educate the impor- 
tance of parental presence in a non-judgmental manner, facilitate a non-punitive environment, and encourage strength-based behaviors.

An APN needs to stay up to date on NAS research. This includes continuing education courses, seminars, conferences, and specialty associations. When the opportunity presents itself, the APN should publish findings based on their research.

The last implication for the APN is to help move policy changes forward. This includes legislating the community's needs, interrupting the stigma of addiction, and ensuring safe care for the mother-infant dyad. APN's need to humanize opioid-addicted mothers to increase awareness and confirm progress in public health policies.

\section{"Clear consensus regarding hospital policies and symptom relief would help many caregivers. In the meantime, help is needed by all to humanize addiction and gain control over the opioid epidemic."}

\section{Conclusion:}

There is still much work to be done regarding infants experiencing NAS. However, defining, screening, testing, assessing, and treating is still a top priority for any provider. As new research is discovered, APN's will need to adjust accordingly for the benefit of the newborn. Clear consensus regarding hospital policies and symptom relief would help many caregivers. In the meantime, help is needed by all to humanize addiction and gain control over the opioid epidemic.

\section{References:}

1. Grossman M, Berkwitt A. Neonatal abstinence syndrome. Semin Perinatol [Internet]. 2019 Apr [cited 2021 Apr 8];43(3):173-186. Available from: https://doi.org/10.1053/j. semperi.2019.01.007

2. Anbalagan S, Mendez MD. Neonatal abstinence syndrome [Internet]. Treasure Island (FL): StatPearls Publishing; 2019 Dec 20 [updated 2021 Jul 21; cited 2021 Apr 8]. Available from: https://europepmc.org/article/NBK/nbk551498\#freefull-text 2019

3. Data and statistics about opioid use during pregnancy [Internet]. Atlanta (GA): Centers for Disease Control and Prevention; 2020 Aug 13 [updated 2021 Jul 21; cited 2021 Apr 8]. Available from: https://www.cdc.gov/pregnancy/opioids/ data.html

4. Martin RJ, Fanaroff AA, Walsh MC. Fanaroff and Martin's neonatal-perinatal medicine; Diseases of the fetus and infant. 10 $0^{\text {th }}$ ed. St. Louis: Elsevier; 2015. 685-694 p.

5. Whalen B, Holmes A, Blythe S. Models of care for neonatal abstinence syndrome: What works? (2019). Semin Fetal Neonatal Med [Internet]. 2019 Mar 26 [cited 2021 Apr 8];24(2):121-132. Available from: https://doi.org/10.1016/j. siny.2019.01.004

6. Palla MR, Khan G, Haghighat ZM, Bada H. EEG findings in infants with neonatal abstinence syndrome presenting with clinical seizures. Front Pediatr [Internet]. 2019 Mar 29 [cited
2021 Apr 8];7:111. Available from: https://www.frontiersin. org/articles/10.3389/fped.2019.00111/full\#B4

7. Grisham LM, Stephen MM, Coykendall MR, Kane MF, Maurer JA, Bader MY. Eat, sleep, console approach. A familycentered model for the treatment of neonatal abstinence syndrome. Adv Neonatal Care [Internet]. 2019 Apr [cited 2021 Apr 8];19(2):138-144. Available from: https://pubmed. ncbi.nlm.nih.gov/30855311/

Conflicts of Interest: The author has no conflicts of interest relevant to this article to disclose.

\section{NT}

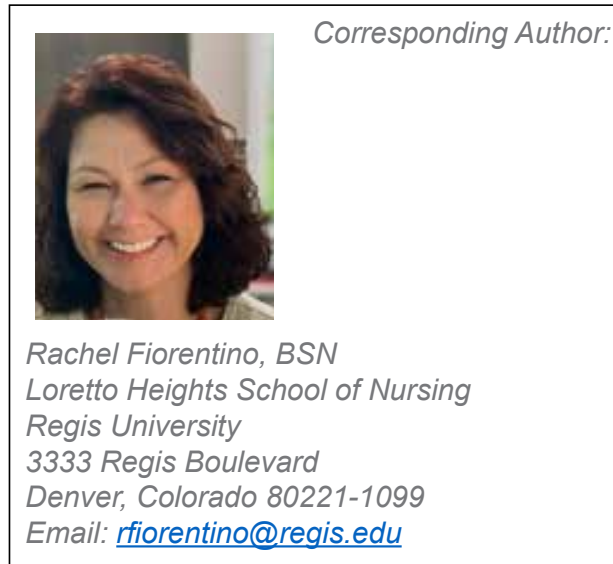

Fellow's Column is published monthly.

- Submission guidelines for "Fellow's Column":

- 2000 word limit not including references or title page. Exceptions will be made on a case by case basis

- $\quad$ QI/QA work, case studies, or a poster from a scientific meeting may be submitted..

- Submission should be from a medical student, resident, fellow, or NNP in training.

- Topics may include Perinatology, Neonatology, and Younger Pediatric patients.

- No more than 20 references.

- Please send your submissions to:

Elba Fayard, MD, Interim Fellowship Column Editor or Japmeet Sandhu, OMS III, Fellowship Column Assistant Editor LomaLindaPublishingCompany@gmail.com 